\title{
UMA ABORDAGEM PROBABILÍSTICA PARA A ESTIMATIVA DA RESPOSTA DINÂMICA DE PASSARELAS MISTAS (AÇO-CONCRETO)
}

\author{
J. M. dos S. de Souza ${ }^{1}$, J. G. Santos da Silva ${ }^{2}$ e L. R. O. de Lima ${ }^{2}$
}

${ }^{1}$ Programa de Pós-Graduação em Engenharia Civil, PGECIV, Universidade do Estado do Rio de Janeiro, UERJ (jorgebulk@hotmail.com)

${ }^{2}$ Departamento de Estruturas e Fundações, Universidade do Estado do Rio de Janeiro, UERJ

Resumo. Passarelas de pedestres com arquitetura moderna, esbeltas e leves são uma constante nos dias atuais, apresentando grandes vãos e novos materiais. Este arrojo arquitetônico tem gerado inúmeros problemas de vibrações excessivas, especialmente sobre passarelas mistas (aço-concreto). As normas e recomendações de projeto consideram, ainda, que as forças induzidas pelo caminhar humano são determinísticas. Todavia, o caminhar humano e as respectivas forças dinâmicas geradas apresentam comportamento randômico. Deste modo, o presente trabalho de pesquisa objetiva contribuir com os projetistas estruturais, a partir do emprego de uma abordagem probabilística para avaliação do estado limite de utilização deste tipo de estrutura, associado a vibrações excessivas que podem vir a causar desconforto humano. Para tal, utiliza-se como modelo estrutural base uma passarela interna construída no novo campus do Instituto de Traumatologia e Ortopedia - INTO, na cidade do Rio de Janeiro. Com base na utilização de métodos probabilísticos, torna-se possível determinar a probabilidade dos valores das acelerações de pico da estrutura ultrapassarem ou não os critérios de conforto humano estabelecidos em normas e recomendações de projeto.

Palavras-chave: Passarelas de pedestres, Dinâmica estrutural, Métodos probabilísticos.

\section{INTRODUÇÃO}

Considerando-se o avanço dos materiais empregados na construção civil, as estruturas estão cada vez mais leves e esbeltas e, portanto, mais flexíveis, tornando o seu comportamento dinâmico cada vez mais crítico na ótica do conforto humano dos usuários. Além disso, a diminuição do amortecimento estrutural e a mudança na natureza e na intensidade das cargas contribuem para esse novo cenário [1-7]. De forma geral, os projetos de passarelas são elaborados considerando, somente, devido à ação de cargas estáticas, não levando em consideração as ações dinâmicas oriundas do movimento de pessoas. Com isso, cada vez mais se tem observado um número crescente de estruturas que apresentam comportamento dinâmico insatisfatório, gerando, portanto, problemas associados ao conforto humano dos usuários [1-7]. 
As normas e guias de projetos [8,9] que tratam dos problemas de conforto humano devido à ação humana consideram as forças induzidas pelo caminhar de pedestres como sendo um processo determinístico. Contudo, em função da variação dos parâmetros da força dinâmica induzida pelo caminhar, tais como peso do pedestre, frequência do passo, comprimento do passo, coeficientes dinâmicos, etc., tal fenômeno apresenta comportamento randômico [1].

Por outro lado, atualmente, os projetistas estruturais têm tido acesso a ferramentas numérico-computacionais com elevado nível de precisão, para estudar o comportamento de estruturas sujeitas a ações dinâmicas, de forma a identificar, ainda durante a fase de projeto, o desempenho da estrutura quando submetida a cargas dinâmicas. Entretanto, o custo computacional para elaboração de um espectro de resposta probabilístico a partir da modelagem computacional é elevado tornando por vezes tal procedimento inviável na prática de projeto [1].

Deste modo, a presente investigação objetiva o desenvolvimento de uma metodologia de análise para avaliação da resposta dinâmica de passarelas de pedestres, mediante o emprego de métodos probabilísticos, no que tange ao conforto humano dos usuários da estrutura. Para tal, utiliza-se como modelo estrutural base uma passarela interna construída no novo campus do Instituto de Traumatologia e Ortopedia - INTO, na cidade do Rio de Janeiro.

Com base no emprego de métodos probabilísticos torna-se possível determinar a probabilidade dos valores das acelerações máximas da estrutura ultrapassar ou não os critérios de conforto humano estabelecidos em normas e recomendações de projeto [8,9]. Considerandose o desenvolvimento da metodologia de análise proposta foi possível determinar os valores das acelerações de pico (acelerações máximas) para uma determinada probabilidade de ocorrência, visando determinar se a resposta dinâmica do modelo investigado é satisfatória ou não, no que tange ao conforto humano dos pedestres.

\section{AÇÕES DINÂMICAS DEVIDO AO CAMINHAR HUMANO.}

De forma geral, a função representativa do caminhar humano tem sido representada por uma série de Fourier, com base em uma composição de harmônicos. Tal representação tem sido utilizada correntemente por diversos autores que estudam este assunto [1-8]. Assim sendo, a representação matemática da ação humana do caminhar (ação dinâmica vertical) é dada pela Eq. (1).

$$
F(t)=P\left[1+\sum \alpha_{i} \cos \left(2 \pi i f_{p} t+\phi_{i}\right]\right.
$$

Onde:

$F(t)$ : função do carregamento dinâmico $(\mathrm{N})$;

$P \quad:$ peso do pedestre $(\mathrm{N})$

$\alpha_{i} \quad$ : coeficiente dinâmico para a força harmônica;

$i$ : múltiplo do harmônico (1, 2, 3, etc.);

$f_{p}$ : frequência do passo do pedestre $(\mathrm{Hz})$;

$t \quad$ : tempo (s);

$\phi_{i} \quad$ : ângulo de fase para o harmônico i (radianos); 
O modelo de carregamento dinâmico utilizado nesta investigação baseia-se em uma análise mais realista para representar a excitação dinâmica proveniente do pedestre, de forma a incorporar o impacto transiente do calcanhar humano. $\mathrm{O}$ modelo matemático adotado no presente estudo foi proposto a partir de uma aproximação respaldada por estudos experimentais que permitiram registrar a reação total de um piso, gerada ao longo do tempo, durante uma caminhada [3], de acordo com as Eqs. (2) a (5).

$$
F(t)= \begin{cases}\left(\frac{f_{m i} F_{m}-P}{0,04 T_{p}}\right) t+P & \text { se } 0 \leq t<0,04 T_{p} \\ f_{m i} F_{m}\left[\frac{C_{1}\left(t-0,04 T_{p}\right)}{0,02 T_{p}}+1\right] & \text { se } 0,04 T_{p} \leq t<0,06 T_{p} \\ F_{m} & \text { se } 0,06 T_{p} \leq t<0,15 T_{p} \\ P+\sum_{i=1}^{n h} P \alpha \operatorname{sen}\left[2 \pi i f_{p}\left(t+0,1 T_{p}\right)+\phi_{i}\right] & \text { se } 0,15 T_{p} \leq t<0,90 T_{p}\end{cases}
$$

Onde:

$F_{m}$ : valor máximo da série de Fourier, dado pela Eq. (3);

$f_{m i}$ : fator de majoração do impacto do calcanhar, $\left(f_{m i}=1,12\right)$;

$T_{p}$ : período do passo;

$C_{1}$ : coeficiente dado pela Eq. (4);

$C_{2}$ : coeficiente dado pela Eq. (5).

A função matemática utilizada para representar a ação produzida por uma pessoa caminhando, não é simulada simplesmente por uma série de Fourier, pois as equações incorporam na formulação o pico transiente representativo do impacto do calcanhar humano sobre a estrutura, como sendo igual a $1,12\left(f_{m i}=1,12\right)$ [3]. Os coeficientes dinâmicos associados aos harmônicos da caminhada são fornecidos pelo guia de projeto do AISC [8], Tabela 1. 
Tabela 1. Coeficientes dinâmicos [10].

\begin{tabular}{ccc}
\hline Harmônico $i$ & Coeficiente Dinâmico $\left(\alpha_{i}\right)$ & Ângulo de Fase $\left(\Phi_{i}\right)$ \\
\hline 1 & 0,5 & 0 \\
2 & 0,2 & $\pi / 2$ \\
3 & 0,1 & $\pi$ \\
4 & 0,05 & $3 \pi / 2$ \\
\hline
\end{tabular}

Na sequência do estudo, a Fig. 1 apresenta uma função de carregamento dinâmico, obtida com base no emprego das Eqs. (2) a (5), associada à ação dinâmica humana vertical, referente a um pedestre caminhando com frequência do passo igual a $2 \mathrm{~Hz}\left(f_{p}=2 \mathrm{~Hz}\right)$.

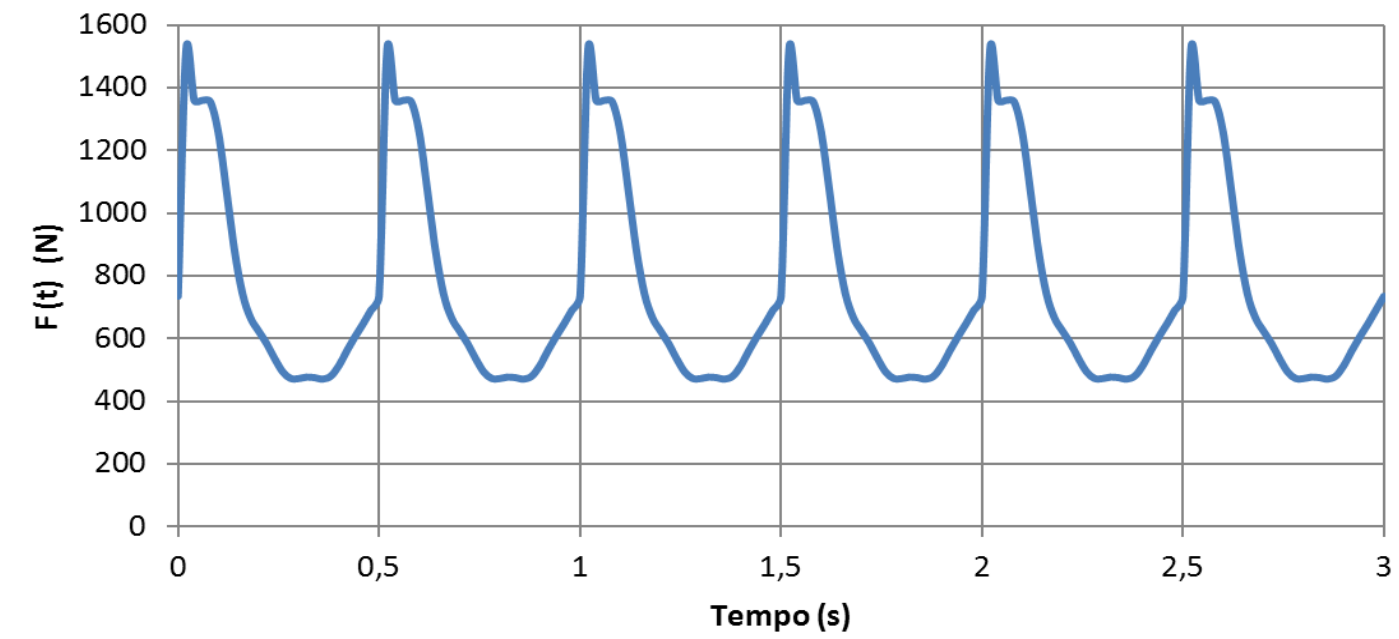

Figura 1. Função de carregamento dinâmico devido ao caminhar de um pedestre $\left(f_{p}=2 \mathrm{~Hz}\right)$.

\section{SOLUÇÕES ANALÍTICAS PARA CÁLCULO DAS ACELERAÇÕES DE PICO}

As expressões analíticas utilizadas neste trabalho de pesquisa consideram as passarelas de pedestres como representadas por sistemas com apenas um grau de liberdade (S1GL). Deste modo, sabe-se que o comportamento dinâmico de um S1GL para um determinado modo de vibração genérico $i$ pode ser representado pela Eq. (6) $[4,11]$.

$$
a(t)+2 \xi_{i} \omega_{i} v(t)+\omega_{i}^{2} u(t)=\frac{\phi_{i} F(t)}{m_{i}}
$$

Onde:
$a(t) \quad$ : aceleração $\left(\mathrm{m} / \mathrm{s}^{2}\right)$;
$v(t) \quad$ : velocidade $(\mathrm{m} / \mathrm{s})$
$u(t) \quad$ : deslocamento $(\mathrm{m})$
$\xi_{i} \quad$ : coeficiente de amortecimento modal;
$m_{i} \quad$ : massa modal do harmônico i;
$\omega_{i} \quad$ : frequência angular da excitação para o harmônico i ( $\left.\mathrm{rad} / \mathrm{s}\right)$;
$\phi_{i} \quad$ : função do modo de vibração i;
$F(t)$ : função do carregamento dinâmico $(\mathrm{N})$. 
Sendo $F(t)$ uma função harmônica do tipo $F(t)=F_{0}$ sen $(\omega t)$, o deslocamento e a aceleração, associados à fase permanente da resposta do sistema (S1GL), podem ser obtidos pelas Eqs. (7) e (8), respectivamente [4,11].

$$
\begin{gathered}
u(t)=\frac{1}{2 \xi_{i} \omega_{i}^{2}} \frac{\phi_{i} F_{0}}{m_{i}} \cos \left(\omega_{i} t\right) \\
a(t)=\frac{1}{2 \xi_{i}} \frac{\phi_{i} F_{0}}{m_{i}} \cos \left(\omega_{i} t\right)
\end{gathered}
$$

\subsection{Método analítico proposto por Rainer et al. [7]}

Rainer et al. [7] propôs um método analítico para o cálculo da aceleração de pico no meio do vão de passarelas. As passarelas são modeladas com sendo um sistema massa-mola com um grau de liberdade (S1GL). A amplitude da força dinâmica devido ao caminhar humano corresponde a $F=\alpha P(\alpha$ : o coeficiente dinâmico e $P$ : o peso do pedestre $)$. Assim sendo, a aceleração da estrutura é calculada com base no emprego do fator de amplificação dinâmico, $\phi$, obtido graficamente para passarelas biapoiadas [7]. Deste modo, a aceleração de pico devido à frequência do caminhar ressonante com a passarela é calculada por meio da Eq. (12) [7].

$$
a=\left(2 \pi f_{n}\right)^{2} \frac{\alpha P \phi}{K}
$$

Onde:

$a$ : aceleração de pico o meio do vão $\left(\mathrm{m} / \mathrm{s}^{2}\right)$;

$f_{n} \quad$ : primeira frequência natural da passarela $(\mathrm{Hz})$;

$\alpha \quad$ : coeficiente dinâmico;

$K \quad$ : rigidez da estrutura $(\mathrm{N} / \mathrm{m})$.

Observando-se a Eq. (9), destaca-se que o valor $\alpha P / K$ representa o deslocamento estático no meio do vão da passarela devido à amplitude da força dinâmica. A aceleração de pico no meio do vão da estrutura pode ser calculada pela Eq. (10), substituindo-se o valor de $(2 \pi f)^{2}$ $=K / m$ [7]. Para passarelas biapoiadas a massa equivalente do sistema é da ordem de metade da massa total da estrutura $(m=17 / 35=0,5 M)$. Deste modo, a aceleração de pico da passarela pode ser calculada também pela Eq.(11) [7].

$$
\begin{aligned}
& a=\frac{\alpha P \phi}{m} \\
& a=\frac{2 \alpha P \phi}{M}
\end{aligned}
$$

Onde:

$m$ : massa equivalente do sistema S1GL $(\mathrm{kg}) ;$

$M \quad$ : massa total do vão $(\mathrm{kg})$. 


\subsection{Método analítico proposto por Murray et al. [8]}

Murray et al. [8] propôs no guia de projeto do AISC um método analítico para o cálculo da aceleração de pico no meio o vão de passarelas de pedestres por meio da Eq.(12).

$$
a=\frac{R \alpha P}{\beta W} \cos \left(2 \pi i f_{p} t\right)
$$

Onde:

$R \quad$ : fator de redução;

$\alpha \quad$ : coeficiente dinâmico;

$P \quad$ : peso do pedestre $(\mathrm{N})$;

$f_{p} \quad$ : frequência do passo do pedestre $(\mathrm{Hz})$;

$\beta \quad$ : coeficiente de amortecimento estrutural;

$W \quad$ : peso efetivo da passarela (kgf);

$t \quad:$ tempo (s).

O fator de redução $R$ leva em consideração que o pedestre caminhando sobre a estrutura e a pessoa perturbada pela vibração oriunda do caminhar nem sempre se encontra no local de maior amplitude modal. $\mathrm{O}$ fator de redução $R$ sugerido para passarelas é igual a 0,7 [8].

\subsection{Método analítico probabilístico proposto por Zivanovic [2]}

Zivanovic [2] propôs um método analítico para o cálculo da aceleração de pico de passarelas adotando-se, basicamente, a mesma formulação presente no guia de projeto do Sétra [4]. Deste modo, a aceleração de pico associada ao primeiro modo de vibração da estrutura pode ser calculada por meio da Eq. (13).

$$
a(t)=\frac{1}{2 \xi m} \alpha P \operatorname{sen}\left(2 \pi f_{p} t\right) \operatorname{sen}\left(\frac{\pi f_{p} l_{p}}{L} t\right)
$$

Onde:

$a(t) \quad$ : aceleração de pico $\left(\mathrm{m} / \mathrm{s}^{2}\right)$

$\xi \quad$ : coeficiente de amortecimento;

$m$ : massa modal da passarela $(\mathrm{kg})$;

$\alpha \quad$ : coeficiente dinâmico;

$P \quad$ : peso do pedestre $(\mathrm{N})$;

$f_{p} \quad$ : frequência do passo $(\mathrm{Hz})$;

$l_{p} \quad$ : comprimento do passo $(\mathrm{m})$;

$L \quad$ : comprimento da passarela (m). 


\section{MODELO ESTRUTURAL.}

O modelo estrutural investigado neste trabalho de pesquisa consiste em uma passarela de pedestre mista (aço-concreto) com vão de 15m, conforme ilustrado na Fig. 2. Os perfis metálicos ASTM A36 utilizados são do tipo "I" soldados. Para as vigas de aço foi considerado um módulo de elasticidade igual a $205 \mathrm{GPa}$. A laje de concreto possui espessura de $0,10 \mathrm{~m}$, resistência característica a compressão de $25 \mathrm{MPa}$ e módulo de elasticidade igual a $28 \mathrm{GPa}$. A Tabela 1 ilustra as propriedades geométricas dos perfis das longarinas e transversinas.

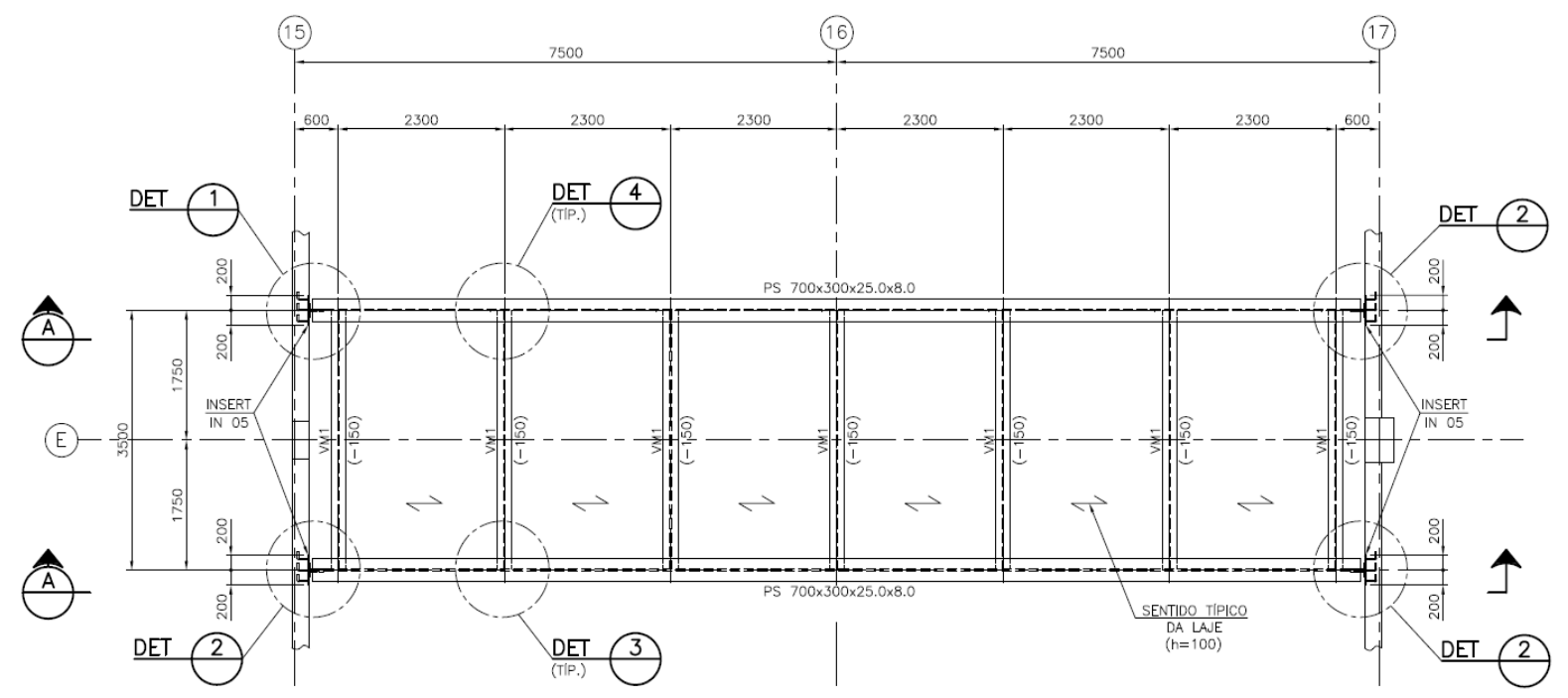

a) Vista superior do modelo estrutural.
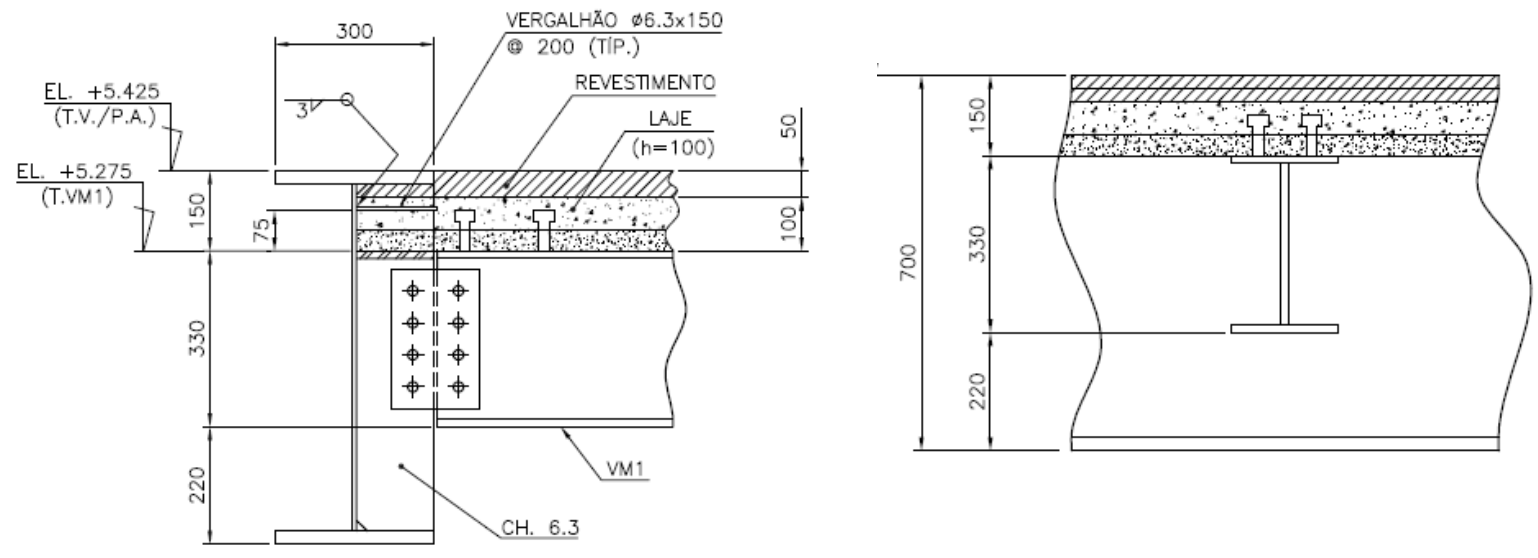

b) Seção transversal da passarela.

Figura 2. Modelo estrutural investigado.

Tabela 2. Características geométricas das vigas da passarela (dimensões em mm).

\begin{tabular}{lccccc}
\hline Tipo de Perfil & $\begin{array}{c}\text { Altura do } \\
\text { Perfil } \\
(H)\end{array}$ & $\begin{array}{c}\text { Largura } \\
\text { da Mesa } \\
\left(b_{f}\right)\end{array}$ & $\begin{array}{c}\text { Espessura } \\
\text { da Mesa } \\
\text { Superior }\left(t_{f}\right)\end{array}$ & $\begin{array}{c}\text { Espessura } \\
\text { da Mesa } \\
\text { Inferior }\left(t_{f}\right)\end{array}$ & $\begin{array}{c}\text { Espessura } \\
\text { da Alma } \\
\left(t_{w}\right)\end{array}$ \\
\hline Longarina - I 700 x 159,9 & 700 & 300 & 25,0 & 25,0 & 8,0 \\
Transversina - I 330 x 45,4 & 330 & 200 & 9,5 & 9,5 & 6,3 \\
\hline
\end{tabular}




\section{MODELAGEM NUMÉRICA}

Na sequência do estudo foi feita uma comparação entre o valor da frequência fundamental $\left(f_{01}\right)$, do modelo em estudo, obtida analiticamente [8], com aquele advindo da modelagem numérico-computacional, via método dos elementos finitos, por meio da utilização do programa Ansys [12]. Deste modo, a Tabela 3 apresenta os valores das cinco primeiras frequências naturais das passarelas, considerando-se variações feitas sobre o comprimento do vão do modelo original investigado $(L=15 \mathrm{~m})$, de acordo com a Figura 2.

Tabela 3. Frequências naturais das passarelas.

\begin{tabular}{|c|c|c|c|c|c|c|c|}
\hline \multirow{2}{*}{$\begin{array}{l}\text { Vão } \\
(\mathrm{m})\end{array}$} & \multirow{2}{*}{$\begin{array}{c}f_{01}(\mathrm{~Hz}) \\
\text { Murray et al. }[8]\end{array}$} & \multicolumn{5}{|c|}{ Frequências Naturais $(\mathrm{Hz})$ [12] } & \multirow{2}{*}{$\begin{array}{c}\text { Diferenças } \\
(\%) \\
\end{array}$} \\
\hline & & $f_{01}$ & $\mathrm{f}_{02}$ & $\mathrm{f}_{03}$ & $\mathrm{f}_{04}$ & $\mathrm{f}_{05}$ & \\
\hline 5 & 29,11 & 30,65 & 54,05 & 59,96 & 69,84 & 83,16 & $5,3 \%$ \\
\hline 6 & 22,20 & 23,16 & 39,74 & 48,35 & 61,84 & 74,32 & $4,3 \%$ \\
\hline 7 & 17,27 & 17,66 & 30,38 & 39,98 & 53,79 & 68,81 & $2,3 \%$ \\
\hline 8 & 13,48 & 14,00 & 24,80 & 41,88 & 52,37 & 60,29 & $3,8 \%$ \\
\hline 9 & 10,90 & 11,12 & 20,72 & 35,48 & 45,88 & 55,33 & $2,0 \%$ \\
\hline 10 & 8,98 & 9,18 & 17,77 & 30,32 & 40,79 & 50,92 & $2,2 \%$ \\
\hline 11 & 7,61 & 7,62 & 15,77 & 26,96 & 44,95 & 46,07 & $0,1 \%$ \\
\hline 12 & 6,44 & 6,13 & 14,04 & 23,30 & 39,83 & 40,22 & $4,8 \%$ \\
\hline 13 & 5,51 & 5,50 & 12,67 & 12,67 & 20,28 & 34,91 & $0,2 \%$ \\
\hline 14 & 4,77 & 4,76 & 11,56 & 17,79 & 30,96 & 32,62 & $0,2 \%$ \\
\hline 15 & 4,12 & 4,10 & 11,08 & 15,86 & 28,53 & 31,76 & $0,4 \%$ \\
\hline 16 & 3,64 & 3,63 & 10,05 & 14,06 & 25,79 & 28,44 & $0,3 \%$ \\
\hline 17 & 3,23 & 3,22 & 9,38 & 12,54 & 23,56 & 25,68 & $0,3 \%$ \\
\hline 18 & 2,89 & 2,86 & 8,79 & 11,24 & 21,64 & 23,30 & $1,0 \%$ \\
\hline 19 & 2,60 & 2,59 & 8,28 & 10,14 & 19,99 & 21,26 & $0,3 \%$ \\
\hline 20 & 2,35 & 2,34 & 7,83 & 9,19 & 18,61 & 19,41 & $0,3 \%$ \\
\hline
\end{tabular}

A partir dos valores das frequências naturais calculadas numericamente observou-se que a estrutura original $\left(f_{01}=4,10 \mathrm{~Hz}\right.$ e $L=15 \mathrm{~m}$ ), Figura 2, encontra-se em ressonância com o segundo harmônico da carga de excitação devido ao caminhar humano. Ressalta-se, ainda, que além das frequências naturais, os deslocamentos e as acelerações de pico também foram obtidos na seção central do modelo, com base no emprego do modelo de carregamento proposto por Varela [3], mediante simulações numéricas, de acordo com a Tabela 4. 
Estes valores de aceleração de pico obtidos numericamente foram comparados com aqueles associados às soluções analíticas para sistemas com um grau de liberdade (S1GL) $[7,8]$, considerando-se, inicialmente, a aleatoriedade do peso do pedestre caminhando com frequência do passo igual a $2 \mathrm{~Hz}$, coeficientes dinâmicos propostos por Murray et al. [8] e coeficiente de amortecimento estrutural igual a $1 \%[8,10]$.

De acordo com os resultados apresentados na Tabela 4 , foi observado que os valores das acelerações de pico, calculados numericamente, apresentam diferenças em relação aos resultados fornecidos pelos métodos analíticos simplificados (S1GL), propostos por Rainer et al. [7] e Murray et al. [8], sendo que estas diferenças quantitativas são da ordem de 19\% e $14 \%$, respectivamente.

Tabela 4. Comparação entre os valores das acelerações de pico $\mathrm{a}_{\mathrm{p}}\left(\mathrm{m} / \mathrm{s}^{2}\right)$.

\begin{tabular}{|c|c|c|c|}
\hline Peso (N) & $\begin{array}{c}\text { Rainer et al. }[7] \\
\mathrm{a}_{\mathrm{p}}\left(\mathrm{m} / \mathrm{s}^{2}\right)\end{array}$ & $\begin{array}{c}\text { Ansys [12] } \\
a_{p}\left(\mathrm{~m} / \mathrm{s}^{2}\right)\end{array}$ & $\begin{array}{c}\text { Diferenças } \\
(\%)\end{array}$ \\
\hline 755 & 0,39 & 0,48 & $18,66 \%$ \\
\hline 637 & 0,33 & 0,41 & $18,88 \%$ \\
\hline 794 & 0,41 & 0,51 & $18,96 \%$ \\
\hline 598 & 0,31 & 0,38 & $18,96 \%$ \\
\hline 627 & 0,32 & 0,40 & $18,96 \%$ \\
\hline 676 & 0,35 & 0,43 & $18,96 \%$ \\
\hline 872 & 0,45 & 0,56 & $18,96 \%$ \\
\hline 882 & 0,46 & 0,56 & $18,96 \%$ \\
\hline 853 & 0,44 & 0,54 & $18,98 \%$ \\
\hline Peso (N) & $\begin{array}{l}\text { Murray et al. [8] } \\
\qquad a_{\mathrm{p}}\left(\mathrm{m} / \mathrm{s}^{2}\right)\end{array}$ & $\begin{array}{l}\text { Ansys [12] } \\
a_{p}\left(m / s^{2}\right)\end{array}$ & $\begin{array}{c}\text { Diferenças } \\
(\%)\end{array}$ \\
\hline 755 & 0,55 & 0,48 & $13,88 \%$ \\
\hline 637 & 0,46 & 0,41 & $13,57 \%$ \\
\hline 794 & 0,57 & 0,51 & $13,46 \%$ \\
\hline 598 & 0,43 & 0,38 & $13,46 \%$ \\
\hline 627 & 0,45 & 0,40 & $13,46 \%$ \\
\hline 676 & 0,49 & 0,43 & $13,46 \%$ \\
\hline 872 & 0,63 & 0,56 & $13,46 \%$ \\
\hline 882 & 0,64 & 0,56 & $13,46 \%$ \\
\hline 853 & 0,62 & 0,54 & $13,43 \%$ \\
\hline
\end{tabular}




\section{ABORDAGEM PROBABILÍSTICA PARA ESTIMATIVA DA RESPOSTA DINÂ- MICA DA PASSARELA}

A metodologia para análise dinâmica probabilística desenvolvida nesta investigação utiliza os valores das acelerações de pico calculadas analiticamente [2,4,7,8,11], com base na consideração de um grande número de análises representativas do espaço amostral em estudo. Neste trabalho de pesquisa, o número de análises realizadas para geração dos resultados foi de 20000 (Vinte mil).

O coeficiente de amortecimento estrutural foi definido como sendo igual a $1 \%[8,10]$ e, concomitantemente, a aleatoriedade dos parâmetros de projeto relevantes para a resposta dinâmica do modelo estrutural (peso do pedestre, frequência do passo do pedestre, comprimento do passo do pedestre e coeficiente dinâmico devido ao caminhar) foi efetivamente considerada neste estudo, adotando-se para tal os valores de média e desvio padrão de acordo com uma distribuição normal, de acordo com o exposto na Tabela 5.

Tabela 5: Média e desvio padrão das variáveis randômicas do problema em estudo.

\begin{tabular}{ccccc}
\hline Variável & Unidade & Média $(\mu)$ & Desvio Padrão $(\sigma)$ & Referências Utilizadas \\
\hline$M$ & $\mathrm{~kg}$ & 75 & 15 & Pedersen e Frier [5] \\
$f_{p}$ & $\mathrm{~Hz}$ & 2,0 & 0,2 & Bachmann e Ammann [10] \\
$l_{p}$ & $\mathrm{~m}$ & $0,83 \mathrm{e}^{-035 f_{n}}$ & 0,025 & Murray et al. $[8]$ \\
$\alpha_{i}$ & - & 0,71 & 0,071 & Zivanovic [2] \\
\hline
\end{tabular}

Com base nos valores das acelerações de pico foi calculada a função de densidade de probabilidade $(f d p)$ e a função de probabilidade acumulada $\left(P_{c}\right)$, no que diz respeito a variável contínua aceleração de pico. A função densidade de probabilidade $(f d p)$ de uma variável é a função matemática cujo gráfico produz a curva teórica que representa a maneira pela qual a probabilidade total é distribuída em relação à amplitude dos possíveis valores desta variável contínua, ou seja, esta função representa a probabilidade de uma variável assumir um determinado valor ou estar compreendida em um intervalo de valores pré-definido. Por outro lado, a função de densidade acumulada $\left(P_{c}\right)$ associada a cada valor possível da variável a probabilidade deste valor ser menor ou igual ao valor escolhido.

Em seguida, a Fig. 3 apresenta as probabilidades cumulativas para as acelerações de pico calculadas com base no emprego dos métodos analíticos $[2,4,7,8,11]$, considerando-se o coeficiente de amortecimento estrutural igual a $1 \%(\xi=1 \%)$. Deve-se ressaltar que o método sugerido por Zivanovic [2] e, bem como, a formulação clássica teórica, associada à resposta dinâmica de um sistema com um grau de liberdade [4,11], apresentam, basicamente, a mesma formulação matemática e, consequentemente, apresentam os mesmos resultados para as acelerações de pico, conforme ilustrado na Fig. 3.

Observando-se os resultados apresentados na Fig. 3, verifica-se, ainda, que os métodos propostos por Murray et al. [8] e Rainer et al. [7] apresentam resultados distintos em relação àqueles obtidos pela formulação clássica teórica $[4,11]$. Esta divergência se deve ao fato de 
que na formulação de Murray et al. [8] e na formulação de Rainer et al. [7], os valores das acelerações máximas são calculados considerando-se a ação dinâmica do caminhar em ressonância com a estrutura e utilizando-se um fator de redução igual a 0,7 e 0,5 , respectivamente.

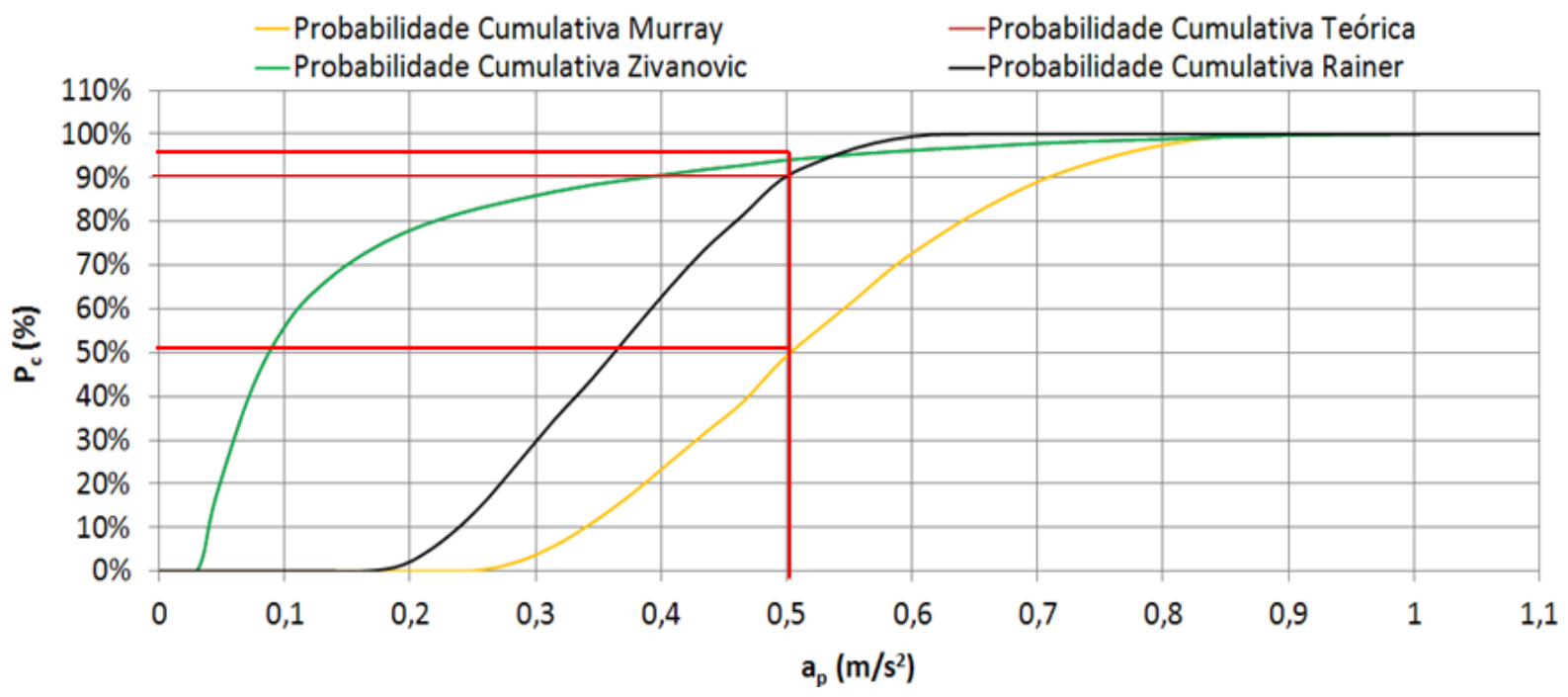

Figura 3. Modelo estrutural investigado.

Considerando-se o valor limite para as acelerações de pico recomendado pela ISO 2631-2 para passarelas de pedestres $\left(a_{l i m}=0,5 \mathrm{~m} / \mathrm{s}^{2}\right.$ [9]), verifica-se, por exemplo, que de acordo com os métodos analíticos propostos por Zivanovic [2], Rainer et al. [7] e Murray et al. [8], a passarela mista (aço-concreto) analisada, ilustrada pela Fig. 2, apresenta acelerações de pico acima do limite normativo em 5\%,10\% e 50\% dos casos, respectivamente, violando os critérios de conforto humano, Fig. 3. Por outro lado, pode-se dizer, também, que para estes mesmos métodos analíticos simplificados [2,7,8], a passarela mista (aço-concreto) investigada encontra-se de acordo com o limite de norma em 95\%, 90\% e 50\% dos casos, respectivamente, Fig. 3, no que diz respeito aos vinte mil casos investigados neste estudo.

\section{CONSIDERAÇÕES FINAIS}

Este trabalho de pesquisa apresenta uma contribuição, no sentido de avaliar a resposta dinâmica de passarela de pedestres, com base no desenvolvimento de uma metodologia de análise probabilística, tendo em mente o emprego de soluções analíticas para sistemas com um grau de liberdade (S1GL). Para tal, um grande número de análises, da ordem de vinte mil, representativas do espaço amostral em estudo foram consideradas. Normas e recomendações de projeto foram utilizadas, no que diz respeito aos valores limites para as acelerações de pico de passarelas de pedestres, objetivando uma análise de conforto humano.

Com o intuito de verificar a influência da aleatoriedade dos parâmetros de projeto (peso, frequência do passo, comprimento do passo e coeficientes dinâmicos) sobre a resposta dinâmica da estrutura, foi verificado que a resposta dinâmica da passarela investigada apresenta valores idênticos quando o comprimento do passo do pedestre é considerado uma variável aleatória ou randômica. 
A partir da metodologia de análise proposta neste estudo foram gerados gráficos com base na variação das acelerações de pico em função da probabilidade acumulativa, para uma passarela mista (aço-concreto) com $15 \mathrm{~m}$ de vão. Com base no emprego destes gráficos foi possível determinar facilmente o valor máximo de aceleração (aceleração de pico) para uma determinada probabilidade de ocorrência no sistema estrutural investigado.

Assim sendo, considerando-se o valor limite para as acelerações de pico recomendado pela ISO 2631-2 para passarelas de pedestres $\left(a_{\text {lim }}=0,5 \mathrm{~m} / \mathrm{s}^{2}\right)$ (ISO 2631-2 1989 [9]), a passarela mista (aço-concreto) investigada neste estudo viola os critérios de conforto humano em 50\% (Murray et al. [8]), 10\% (Rainer et al. [7]) e 5\% (Zivanovic [2]), no que diz respeito aos vinte mil casos analisados.

\section{Agradecimentos}

Os autores deste trabalho de pesquisa agradecem ao suporte financeiro fornecido pelas Agências de Fomento à Pesquisa do país: CAPES, CNPq e FAPERJ.

\section{REFERÊNCIAS}

[1] Souza, J.M. dos S de., Metodologia de análise probabilística para avaliação do comportamento dinâmico de passarelas mistas (aço-concreto). Dissertação de Mestrado, Programa de Pós-Graduação em Engenharia Civil, PGECIV, Universidade do Estado do Rio de Janeiro, UERJ, Rio de Janeiro, Brasil. 2012.

[2] Zivanovic, S., Probability estimation of vibration for pedestrian structures due to walking. PhD Thesis, Department of Civil and Structural Engineering, University of Sheffield, UK. 2006.

[3] Varela, W.D., Modelo teórico-experimental para análises de vibrações induzidas por pessoas caminhando sobre lajes de edificios. Tese de Doutorado, Universidade Federal do Rio de Janeiro, Departamento de Engenharia Civil, COPPE/UFRJ, Rio de Janeiro, Brasil. 2004.

[4] Sétra: Servisse d'études techniques des routes et autoroutes. Assesment of vibrational behaviour of footbridge under pedestrian loading, 131 pgs. 2006.

[5] Pedersen, L. e Frier, C., Sensitivity study of stochastic walking load models, Department of Civil Engineering, Aalborg University, Sweden. 2001.

[6] Hauksson, F., Dynamic behavior of footbridges subjected to pedestrian-induced vibrations. MSc dissertation, Structural Mechanics, Lund University, Sweden. 2005.

[7] Rainer, J.H., Pernica, G. e Allen, D.E., Dynamic loading and response of footbridges. Canadian. Journal of Civil Engineering, v. 15, n. 1, p. 66-71. 1988

[8] Murray, T.M., Allen, D.E. e Ungar, E.E., Floor vibrations due to human activity. Steel Design Guide Series, American Institute of Steel Construction, AISC, 70 pgs. 2003. 
[9] International Organization for Standardization ISO 26231-2. Evaluation of human exposure to whole-body vibration - Part 2: Continuous and shock-induced vibrations in buildings (1 to $80 \mathrm{~Hz}$ ). 1989.

[10] Bachmann, H. e Ammann, W., Vibrations in structures induced by man and machines. Structural Engineering Document 3e, International Association for Bridges and Structural Engineering, USA. 1987.

[11] Clough, R.W. e Penzien, J., Dynamics of Structures. McGraw-Hill, New York, USA, 634 páginas. 2003.

[12] Ansys. Swanson Analysis Systems. Inc., P.O. Box 65, Johnson Road, Houston, PA, 15342-0065, Version 10.0, Basic analysis procedures, Second edition. 2009. 